\title{
Index of topics
}

The main purpose of this index is to amplify and complement the editors' introduction, encouraging readers to pursue themes and ideas encountered in one particular essay across the rest of the volume. It is not a comprehensive index of subjects treated, let alone those only mentioned in passing. It is highly selective, especially because it (mostly) omits the subjects which are historically specific to the different essays and instead highlights the 'words, concepts and things' which form their authors' underlying concerns. 'Words, concepts and things' is a favourite formulation of Susan Reynolds, and the content and arrangement of the index also owe much to the inspiration which the indexer has found in Susan's own books, essays and conversation.

The primary arrangement follows the three broad areas indicated in the main title of the book, adding sections on kinship and family (which might, perhaps, have come under laity) and on words and language. Under the subheadings the arrangement is alphabetical. There are necessarily many connections and overlaps between the different sections.

\section{LAW AND POLITICS}

A PROPERTY RIGHTS AND RELATIONS

B CRIME AND PUNISHMENT

C LEgAL PROCEDURE

D POLITICAL AND LEGAL NORMS

II LAITY

A Lay Values

B NATIONAL IDENTITIES AND

CHARACTERISTICS

C THE LAITY AND THE CHURCH

III KINSHIP AND FAMILY

A KINSHIP SYSTEMS

B PARTICULAR RELATIONSHIPS

C WOMEN

IV SOLIDARITIES AND COLLECTIVITIES

A TYPES OF COLLECTIVITY
B EXPRESSIONS AND ACTIVITIES OF

COLLECTIVITIES

$\mathrm{V}$ WORDS AND LANGUAGE

A MEDIEVAL WORDS

B MEDIEVAl LANGUAGE, LITERACY AND SYMBOLS

C MODERN HISTORIOGRAPHICAL IDEAS

I LAW AND POLITICS

IA PROPERTY RIGHTS AND RELATIONS

property rights in general 18-20, 26

church lands

granted to laymen 17-18

grants by laymen to the church $68-82$, 90-2

leased to laymen 18-19 
communal 181

belonging to lineages $177-8$

disputed 35, 124-7

dower 2, 8, 150-3, 155n.21, 157-8

dowry 49, 51-3, 256

inheritance 2, 8, 196; see also IIA: inheritance

in law codes

barbarian 50-4

ecclesiastical 54-9

late Roman 48-50

in marriage and stepfamilies 48-53

tenure (types)

cornage 154-5

gavelkind 155,157

serjeanty 152

socage $152-3,155 n .21,155$ n.23, 156, 159

\section{IB CRIME AND PUNISHMENT}

crime in general 71, 126, 133, 144, 147, 153

bad counsel (as an offence) 75

homicide 97, 98, 99, 101, 105-6, 111-12, 136, 155n.18, 177

incest 4, 6, 8, 191, 194; see also IIIA: consanguinity; IIIB: marriage, incestuous

perjury 101-5, 109-10, 127

punishment

beheading 153

forfeiture of land 71-2, 73-4, 76, 78-9

murdrum fine 153-4

sexual infidelity and misbehaviour 97, 98, $111,135,136,225-7$

treason 5, 76, 95-115, 116-17, 125-6, 132-3, 134-6, 139-41, 147

\section{IC LEGAL PROCEDURE}

legal procedure in general 84

accords, arbitration and agreements 98, $115,124-5,139-40,145-6,182$, 184

debates in and out of court 3,101, 103-6, 139,183

defiance 105-6, 107, 111

frankpledge 223, 224

jurisdiction

clerical and lay participation in judgement 210

communal judgement 182 judges $3,150,157$

juries 152, 156, 220, 223-4

oaths 100-1, 105

denial in court 97

fidelity 38, 100, 114, 223

jurors' 131

mutual 34, 36-7, 38, 39, 40

at ordeals 130,131

ordeals $6,98,115,118-19,121-2,123-$

$$
\text { 4, 129-30, 142, } 143
$$

ecclesiastical censure 129-30

see also trial by battle

peace-keeping and dispute resolution 4,

101, 177, 178-9, 181-2, 210, 222,

225-6

professional law and lawyers 115, 159, 182,204

treason, procedure for accusation 138

trial by battle (judicial duel) 4, 6, 96, 979, 102, 115, 116-49

ecclesiastical censure 129-30

intervention by a saint $137-8$

jurisdiction over outcome 143

lay censure 130-1

medieval terms 120,136

procedure 128-9, 138-9, 145

trials

actual 113

fictional 95-101, 107, 110, 113-15

ID POLITICAL AND LEGAL NORMS

legal norms

English common law 141-2, 150-9

Law-codes 27, 75

legal education for future rulers 142

local custom 3, 5, 150-9

papal 191-9

Welsh and Irish 174, 178

government and administration 4, 218-33

aldermen 219-20, 222, 223-7, 229-32

councils 74-5

government officials $33,35,85,150-5$, 157-8, 174

kingship see IIA: kingship

politics 116-49, 200-17

political ideas, values and discourse 6 , 68-82, 115, 118, 134, 136, 193-6, 204-5, 213-15 
war and violence 4, 21-2, 24-5, 35, 72

armies and soldiers 13, 16, 18, 40-1, 45

crusades 245-6

foreign conquest $15,165,170-1,174$

hostage-taking and ransom 102-3, 105

military leadership 24, 25, 165, 170

military service $13,17-18$

rebellion 113-14

within the family $15,24-5$

see also IC: trial by battle

\section{LAITY}

\section{A LAY VALUES}

ideals and thought in general 2, 7, 80

attitude to consanguinity 190-1

attitude to relics $238,242-4$

attitude to treason 113

attitude to trial by battle see IC: trial by battle

counsel see IVB: counsel

fidelity 2, 38, 41-3, 95-115, 214-15

honour and shame $6,7,10,107-8,115$, $119,122,135-6,181$

inheritance 77-9; see also IA: inheritance kingship 15, 21, 26, 27-8, 44, 72-3, 80, $82,185,246-7$

kingship as tyranny $165-8,171$

sacral 206-9

laicisation 6; see also VC: modern

historiographical ideas

medieval mentalities 83-4, 87-8

rank 27-8, 44, 46

religious scepticism 2

vertical relationships

dual lordship 110-11, 113

king-subject 28, 42-3, 214

lord-community $181,186-7$

lord-man 102-11, 113-14

lordship in Wales 172-87

II B NATIONAL IDENTITIES AND

CHARACTERISTICS

national identities in general 5, 76

Angevin 165
English 11, 75-6, 160-1, 165-70

Francophobia 168-9, 171

in Ireland 164, 167

in Wales 162-4, 167

Frankish 21-2, 166

French 164-6, 168

in Wales 164

German 166

Norman 168

in England 160-1, 165-70

in Ireland 164-5, 168

in Wales 162-4

Roman 166

Saxon 166

'Trojan' (i.e. Welsh) 162-4

Welsh 162-6, 175

IIC THE LAITY AND THE CHURCH

church lands see IA: church lands

church reform 71-2, 80, 206

false monasteries 33

lay-clerical distinctions and relations 3, $7-$ 8, 10, 29, 200-17, 220, 249-61

religious patronage by kings $68-82,260$

three orders of society $27-8,216$

\section{KINSHIP AND FAMILY}

III A KINSHIP SYSTEMS

chiefs of kin groups 176, 179

clans ('imagined kinship communities') 4, 9, 189, 198, 251, 252

common European kinship system 190-1

consanguinity 190-9; see also IB: incest; III B: marriage, incestuous

differences between regions of Europe 189-90

dynasties 71,82

family piety 76

feud 189

genealogy 178,185

involvement in disputes 113

kin relationships in general 175, 179-80

kinship systems and family in general 4 , $5-6,8-9,9-10$

kinship terminology 177 
political solidarity of Carolingians 15, 22-4, 36, 61-2

provision for royal families 72,74

relations between kin groups and lords 175, 176-7

supposed transformation from cognatic to agnatic $188-9$

surnames 189

Welsh and Irish 173-4, 175-6

youth and maturity $68,71,81$

III B PARTICULAR RELATIONSHIPS

children

care of 47,68

posthumous 48

sons of remarried widows 51

stepchildren 47-67

stepdaughters $49,56,58$

stepsons $51,56,59$

stepsons of widows 52

younger sons 155-6

godparents 197

husband's kin 53

marriage and concubinage 23, 33, 63, 81, 191-9

divorce and annulment 58, 197

endogamous and exogamous 195 , 198-9

incestuous 49, 51-2, 54-7, 59; see also

I B: incest; III A: consanguinity

papal dispensations for marriage 192-7, 199

second and subsequent marriages 48$54,57-9$

paternal uncles 51

stepfathers 48-52, 56, 58, 61

stepmothers $8,47-67$

in legislation 47-59

medieval terminology $47-8$ n.3, 60-2

political influence $60-7$

widowers 49-51, 157-8

widows 48-9, 50, 51-4, 58, 154

property see IA: dower

widow-burning 50

IIIC WOMEN

in general 9-10

abduction 55

in Buddhism 249-50, 252-3, 254, 255-6 chastity 54

clerical attitudes 239-41

control of household property and clothing 60, 63-7, 154

criminals $34-5$

excluded from public sphere 223

guild members 37,39

in Hinduism 261

property rights and inheritance $69,74,77$, 79; see also IA: dower

in ruling families 23-4, 25

\section{SOLIDARITIES AND \\ COLLECTIVITIES}

IVA TYPES OF COLLECTIVITY

solidarities and collectivities in general 1 , $3,4,172,187$

caste 259-60

craft guilds

India 251-2, 256-9

London 220, 223, 224, 232, 233

criminal gangs $4,34-5,37,40,41$

crusade (as a form of lay solidarity) 235,

245-6

cultic 8

family $76-7,176,188-99,211,252-3$, 255

friendship 32-3, 74, 175, 193-5, 198

king's advisers 71,73

neighbourhoods (village) 4, 33-6, 179-83, 186

charitable social provision (fraternities)

4, 36, 37, 38, 40, 210

military self-defence 39,45

peace-keeping associations 210

pilgrimage (as a form of lay solidarity)

235-6, 244-5, 251

regional 3-4, 5, 21-2

regnal 3, 5, 210

English 160, 162, 168

French 205-7, 210-11, 212-13

see also IIB

seminars 11

sociability 183-5; see also IVB: convivial drinking

urban parishes 220, 223, 233 
urban wards $218-33$

attendance 222-3

conduct of meetings and elections 2214, 231

IVB EXPRESSIONS AND ACTIVITIES OF COLLECTIVITIES

agricultural co-operation 4, 175, 178, 180-1

assemblies 28, 33-4, 38, 53, 90, 183-5, 218-33

convivial drinking 4, 36-8, 40, 41, 45, 59 counsel 3, 7, 10, 73-5, 77, 82, 103, 105, $110,113,114,148$

elite formation 20-1

mutual oaths see IC: oaths, mutual parity (as a medieval concept of equality) 29

peers $3-4,27-46$

angels 43

baronial and knightly 4-5, 116-18, 134, 139

bishops 31-2

Charlemagne's twelve 98, 111, 112

combatants injudicial duel 139, 141, 148

counts 28

courtiers 33

elite soldiers 41-2

humanity before God 29-31, 223

inhabitants of London 222-3

lord's men 3, 114

magnates of England 139

mayor and jurati of towns 147

peasant soldiers 41

of the realm (as Carolingian ideal)

$42-5$

servi 36

shared memory 4

\section{WORDS AND LANGUAGE}

VA MEDIEVAL WORDS

aequales, aequaliter 29-31

appellatio, appellator 116, 117, 131, 133

bataille, batalha 120,129

bellum 120, 121, 124, 136, 142 beneficium 18

boni homines 5, 35, 182

certamen 120

conjurado, conspirado 36-9

consortes 33, 36

conspirado see conjurado consuetudo 151-2, 154; see also lex et consuetudo

convenientia 36-8, 40, 42

cultus 30-1

cymydog 181

documentum 89

duellum 116, 120, 127, 128, 131, 136, $138,143,145,147$

equales see aequales

fideles 42

rater laicus 208

gens $31,32,71,75,169$

hereditas $78-9$

judicium 121, 124, 130, 142

laicus see frater laicus

lex et consuetudo 157-8

miles Christi 208

modernitas 86

monomachia 129

mos 152

nacio, natio 71, 73, 76, 179

natura 31

ordo 31

pares (Latin), pairs (French) 28, 30, 32-3, $35,37-8,41,43,45$

patria 10-11, 75-6, 78, 152, 154, 184

placitum 90, 131

populas 71

precaria $17,18-19$

prodido 116-17, 126, 133, 141

proelium 136

progenia 198

secularis 209

societas 43

traïson 111,134

viri sapientes 35

VB MEDIEVAL WRITING, LITERACY AND

SYMBOLS

authentication of documents 89-94

Bible as read and quoted in Middle Ages $29,42,51,55-6,86$ 
bilingual societies 161, 169, 257

classical texts as read and quoted in

Middle Ages 43-5, 86

documentary production and retention 2 , $6,8,20-1,85,88-92,178$

documents as title to land 19-20, 69-70

hagiography $16-17$

history-writing $17,70-1$

number symbolism 28, 191-2

human body and life-stages as metaphor for society $10,68,80-2,211-15$

legends and traditions 12, 16, 22

orality 2, 6, 85, 93

relics 6, 236-8, 241-2, 250

religious satire $235,237-8,244$

ritual 6, 235-6

marriage 53-4

oath-swearing 38, 45

punishment 36

requests for pardon and favour 27 use of vernacular 95-7, 115, 120, 147,

245, 247-8

writing 88-9, 93-4

VC MODERN HISTORIOGRAPHICAL IDEAS

acculturation 5, 13, 20-2, 26, 87, 174, 178-9

'Celtic' obscurantism 173

cultural diffusion 2

the difference of the Middle Ages 2-3, 7, 83-94

English exceptionalism 3, 172-4

'feudalism' 1, 3, 5, 13-14, 17-18, 85-6, 114

French normativism 3, 173-4

progressivism (the idea of progress from 'primitive' to 'modern') 1, 2, 6, 83, 86 belief in growth of laicisation in later

Middle Ages 203-7

modern secularism 201

state-building and political unification 1 